\title{
Resveratrol induces p53 in colorectal cancer through SET7/9
}

\author{
ZHONGLUN LIU ${ }^{1 *}$, XIAOHONG WU ${ }^{2 *}$, JINGJING LV $^{3}$, HUI SUN $^{3}$ and FEIQIN ZHOU ${ }^{4}$ \\ ${ }^{1}$ Department of Clinical Laboratory, The First People's Hospital of Lianyungang, Lianyungang, \\ Jiangsu 222002; ${ }^{2}$ Department of General Surgery, The Affiliated Yixing Hospital of Jiangsu University, \\ Yixing, Jiangsu 214200; ${ }^{3}$ Department of Clinical Comprehensive Experiment Centre, \\ Lianyungang Oriental Hospital, Lianyungang, Jiangsu 222042; ${ }^{4}$ Department of Medical Examination Centre, \\ The Affiliated Yixing Hospital of Jiangsu University, Yixing, Jiangsu 214200, P.R. China
}

Received December 8, 2017; Accepted September 4, 2018

DOI: $10.3892 / 01.2019 .10034$

\begin{abstract}
Resveratrol is one of the most promising phytoalexins for use as an anti-cancer agent, which is present in the skin of red grapes and berries. Resveratrol has been demonstrated to modulate a number of signalling pathways that are involved in carcinogenesis. In the present study, the function of resveratrol as a pro-apoptotic agent in colorectal cancer cell lines, including HCT116, CO115 and SW48, was investigated. The results revealed that resveratrol supressed cell viability. Additionally, resveratrol enhanced the expression of tumour protein p53 (p53) and p53 target genes, including Bcl2 associated $\mathrm{X}$, apoptosis regulator and $\mathrm{Bcl} 2$ binding component 3 that have a pivotal role in p53-dependent apoptosis. Furthermore, treating cells with resveratrol upregulated SET domain containing lysine methyltransferase 7/9 (SET7/9) expression, which positively regulates p53 through its mono-methylation at lysine 372, compared with untreated cells. Furthermore, treating cells with resveratrol induced the expression of apoptotic markers including cleaved caspase-3 and poly (ADP-ribose) polymerases (PARP) compared with untreated cells. However, the genetic knockdown of SET7/9 by short hairpin RNA attenuated the resveratrol-driven overexpression of p53, cleaved caspase-3 and PARP. Collectively, these results reveal the molecular mechanisms by which resveratrol induces p53 stability in colon cancer that results in the activation of p53-mediated apoptosis.
\end{abstract}

\section{Introduction}

Resveratrol (trans-3,4,5-trihydroxystilbene) is a non-flavonoid polyphenolic compound produced naturally in grapes and

Correspondence to: Dr Feiqin Zhou, Department of Medical Examination Centre, The Affiliated Yixing Hospital of Jiangsu University, 75 Tongzhenguan Road, Yixing, Jiangsu 214200, P.R. China E-mail: zhoufeiqin2017@sina.com

*Contributed equally

Key words: resveratrol, colorectal cancer, tumour protein p53, SET domain containing lysine methyltransferase $7 / 9$, apoptosis nuts (1) that is also present in Polygonum cuspidatum root (2). Resveratrol is present in the skin of grapes, and is been used by plants for protection against fungi and other infections (3). However, resveratrol has a number of other properties, including anti-atherogenic, anti-inflammatory and anti-cancer effects (4). Furthermore, previous research has demonstrated that using resveratrol as a chemo-preventive agent results in the inhibition of the cell cycle in tumour cells (5).

Resveratrol has an anti-tumour potential in colorectal cancer (6,7). In 2017 colorectal malignancy was the third most common cancer type in men and women in the United States that comprises $\sim 9 \%$ of all cancer types (8). Colorectal cancer development may be divided into two main phases, the initiation phase and the progression phase. The initiation phase includes the inactivation of the tumour suppressor gene adenomatous polyposis coli, which controls various cellular processes, including cell division and proliferation $(6,9)$. In addition, $\beta$-catenin is translocated from the cytoplasm to the nucleus through the Wnt signalling pathway, in which $\beta$-catenin functions as an oncogene. The progression phase is mostly characterised by the genomic instability of several proteins including K-Ras, SMAD family member 4 and tumour protein p53 (p53) that results in colorectal carcinoma progression (10).

p53 is a tumour suppressor that orchestrates the cellular responses to genotoxic stresses (11-13). Activation of p53 induces the transcription of its target genes that are involved in cell cycle arrest, apoptosis and DNA repair (14). p53 facilitates apoptosis through transactivating a number of p53-target genes including $\mathrm{Bcl} 2$ associated $\mathrm{X}$, apoptosis regulator (BAX) and $\mathrm{Bcl} 2$ binding component 3 (PUMA) (15). p53 is mainly regulated through various post-translational modifications (PTMs), including acetylation, methylation, neddylation, phosphorylation and ubiquitination $(13,16)$. Previous studies have demonstrated the involvement of resveratrol in the cellular function of p53 (17-20). Furthermore, the pro-apoptotic activity of resveratrol in prostate cancer appeared to be mediated by the serine-15 phosphorylation of p53 by mitogen-activated protein kinases (21). However, the effect of resveratrol on other PTMs of p53 has not been investigated in colorectal cancer. SET domain containing lysine methyltransferase 7/9 (SET7/9) is one of the proteins that regulate p53 through PTMs, which mono-methylates p53 at lysine 372 (K372) that results in its stabilization and activation $(11,16)$. 
In the present study, the investigated the mechanisms underlying the impact of resveratrol in p53 activation. To address this question, we examined SET7/9 as a mediator of Resveratrol-dependent p53 activation. The results of the present study provide the mechanistic explanation for the regulatory function of resveratrol in the p53 pathway.

\section{Materials and methods}

Plasmid constructs. Scrambled (cat no. shc016: CCGGGCGCG ATAGCGCTAATAATTTCTCGAGAAATTATTAGCGCT ATCGCGCTTTTT) andSET7/9 (cat no.SHCLND-NM_030648: CCGGGCCAGGGTATTATTATAGAATCTCGAGATTCT ATAATA ATACCCTGGCTTTTTG) short hairpin RNA (shRNA) were obtained from Sigma-Aldrich (Merck KGaA, Darmstadt, Germany). $\beta$-galactosidase (cat no. RC200721; Origene Technologies, Inc., Rockville, MD, USA), pGL3-basic vector constructs were obtained from Promega Corporation (cat no. E1751; Madison, WI, USA) and BAX or PUMA promoter regions were synthesized by GenScript (Piscataway, NJ, USA).

Cell culture and plasmids. All cell lines used in the present study (HCT-116, CO-115 and SW48) purchased from the American Type Culture collection (Manassas, VA, USA) were cultured in Dulbecco's modified Eagle's medium supplemented with $10 \%$ foetal bovine serum (Thermo Fisher Scientific, Inc., Waltham, MA, USA) at $37^{\circ} \mathrm{C}$ with $5 \% \mathrm{CO}_{2}$. The 293 cells were seeded into 24 -well culture plates $\left(3.5 \times 10^{-4}\right.$ cells $\left./ \mathrm{ml}\right)$ and changed the medium without FBS following an overnight incubation at $37^{\circ} \mathrm{C}$ with $5 \% \mathrm{CO}_{2}$. Transfections were performed using Lipofectamine 2000 reagent (Invitrogen; Thermo Fisher Scientific, Inc.) according to the manufacturer's protocols. Approximately 18-24 h prior to transfection, plate cells in $1 \mathrm{ml}$ complete growth medium per well in a 12-well plate. Cells were 70-90\% confluent at the time of transfection. Cells were plated at a density of $1 \times 10^{5}$ cells/well and incubated at $37^{\circ} \mathrm{C}$ overnight. The medium was replaced with $1 \mathrm{ml}$ of fresh complete growth medium and $100 \mu \mathrm{l}$ of serum-free medium was placed in a sterile tube. A total of $1 \mu \mathrm{g}$ plasmid DNA was added to the medium in the tube and mixed completely by gently pipetting up and down. A total of $2 \mu \mathrm{l}$ transfection reagent was added to the diluted DNA mixture and mixed. The samples was incubated at room temperature for $20 \mathrm{~min}$ to allow sufficient time for complexes to form. The supernatant of medium was collected to infect the cancer cells. The viral titer was determined by Virus drops degree detection; fluorescence/absolute quantitative method (17). The cancer cells were infected by the collected supernatant for $48 \mathrm{~h}$ and collected for Western blot analysis. shRNA transfection was performed directly, without using Lentivirus. Resveratrol or dimethyl sulfoxide (DMSO) were obtained from Sigma-Aldrich (Merck $\mathrm{KGaA})$.

Cell viability. A total of 10,000 (HCT-116, CO-115 and SW480) cells were cultured in 96-well plates. After $24 \mathrm{~h}$, the cells were treated with various doses $(0,12.5,25,37.5,50 \mu \mathrm{M})$ of resveratrol (cat no. 34092-100 MG; Sigma-Aldrich, Merck KGaA, Darmstadt, Germany) and incubated for $24 \mathrm{~h}$ at $37^{\circ} \mathrm{C}$. Cell viability was measured using a CellTiter-Glo Luminescent cell viability assay (cat no. G7572; Promega Corporation) with the
Glomax Explorer Microplate Reader (Promega Corporation) according to the manufacturer's protocols.

Western blotting. The protein was extracted from the HCT-116, CO-115 and SW480 cells with $10 \%$ MSDS (Sigma-Aldrich; Merck KGaA, Darmstadt, Germany) and the protein quantity was determined using a bicinchoninic acid assay. The mass of the proteins loaded in per lane was $60 \mu \mathrm{g}$. The proteins were separated using 10\% SDS-PAGE, and then transferred onto polyvinylidene fluoride membranes. The membranes were blocked in $5 \%$ bovine serum albumin (cat. no. 10099141; Gibco; Thermo Fisher Scientific, Inc.) at $37^{\circ} \mathrm{C}$ for $1 \mathrm{~h}$, and washed with TBST buffer three times. The membranes were exposed to primary antibodies $(1: 1,000$ dilution): p53 (Merck KGaA), methylated-p53 (K372; cat no. ab16033; Abcam, Cambridge, UK), SET7/9 (cat no. 2813) and poly (ADP-ribose) polymerase (PARP; cat no. 9542S; Cell Signalling Technology, Inc., Danvers, MA, USA) and $\beta$-actin (cat no. ab8227; Abcam). overnight at $4^{\circ} \mathrm{C}$. The membranes were washed with TBST three times, and incubated with secondary antibody (anti-rabbit Immunoglobulin G; 1:2,000; cat. no. 7074; CST) for $2 \mathrm{~h}$ at room temperature, and washed with TBST three times. The bands were visualized with the WEST ZOL Plus system (iNtRON Biotechnology, Boston, MA, USA) and quantified using ImageJ software (version 1.44P; National Institutes of Health, Bethesda, MA, USA).

Luciferase assay. The luciferase assay was performed as previously described (12). Briefly, 100,000 HCT-116 or CO-115 cells per well were cultured in 24-well plates. After $24 \mathrm{~h}$, the cells were transfected with luciferase genes (pGL3-PUMA or pGL3-BAX or pGL3 vectors) and $\beta$-galactosidase constructs using Lipofectamine 2000 at $37^{\circ} \mathrm{C}$ with $5 \% \mathrm{CO}_{2}$. The following day, the RPMI-1640 medium supplemented with 10\% FBS media was replaced by fresh RPMI-1640 (with 10\% FBS) growth media and 6 samples were treated with different resveratrol doses $(0,12.5,25,37.5,50 \mu \mathrm{M})$ at $37^{\circ} \mathrm{C}$. A total of $24 \mathrm{~h}$ later, the lysates were collected in lysis buffer (within the kit) and stored at $-80^{\circ} \mathrm{C}$ for $3 \mathrm{~h}$; and the luciferase signals were assessed using a luminometer using a Luciferase kit (cat no. K801-200; BioVision, Inc., Milpitas, CA, USA) according to the manufacturer's protocol.

Bioinformatics analysis. The publicly available bioinformatics data set (GSE17537: 244653_at) was downloaded and analysed. The bioinformatics analyses were obtained from PrognoScan $(22,23)$ (http://www.abren.net/PrognoScan). 55 patients with colorectal cancer (CRC), included 30 male and 25 female, with a median age of 62 years old $($ mean \pm SD 62.23 \pm 3.43 , range 43-76). Tumor stage was determined according to the tumor-node-metastasis (TNM) classification system of the International Union against Cancer (2002). The tumors' differentiation have been graded by the Edmondson-Steiner classification system. The clinical pathological features of the patients are summarized in Table I.

Statistical analyses. Data were compiled in Microsoft Excel 2011 14.7.2 (Microsoft Corporation, Redmond, WA, USA) and the evaluation of variance between data groups was performed using a one-way analysis of variance. If significance 
was detected further comparisons were performed with a Tukey's post-hoc test. All data are presented as means \pm standard deviation (SD) or median with $95 \%$ confidence interval (95\% CI). $\mathrm{P}<0.05$ was considered to indicate a statistically significant difference. Kaplan-Meier survival curves with log-rank tests were used in the bioinformatics data analysis.

\section{Results}

Resveratrol induces SET7/9 expression in colorectal cancer cells. The expression of SET7/9 is associated with tumorigenesis in different cancer types, including gastric (24), breast (25) and colorectal cancer. To understand the association between SET7/9 expression and the survival rate of patients with colorectal cancer, the publicly available bioinformatics data set (GSE17537: 244653_at) was analysed, and the results suggested a significant positive association between a low overall survival rate and the downregulation of SET7/9 $(\mathrm{P}=0.011$; Fig. 1A). Resveratrol has been suggested as an anti-cancer agent in colorectal cancer (7), which induces the expression of p53 (18). Therefore, it was hypothesized that resveratrol may regulate p53 through the induction of SET7/9 expression in colorectal cancer cells. To address this, initially the concentration $(0,12.5,25,37.5,50 \mu \mathrm{M})$ of resveratrol that promoted cell death in HCT116, CO115 and SW48 cell lines was determined (Fig. 1B). Then, the expression of SET7/9 in response to resveratrol treatment was assessed. As presented in Fig. 1C-E, the cells treated with resveratrol demonstrated higher protein expression of SET7/9 compared with those that were treated with DMSO. Collectively, these data indicate that resveratrol is a positive regulator of SET7/9.

SET7/9 mediates resveratrol-dependent p53 overexpression. Mono-methylation of p53 at K372 by SET7/9 has a key role in p53 stability and activity (16). Therefore, the present study determined the protein expression of methylated p53 K372 $\left(\mathrm{p} 53^{\mathrm{K} 372-\mathrm{Me}}\right)$ in the presence of resveratrol or DMSO (Fig. 2A-C). The results revealed a higher protein expression of $\mathrm{p} 53^{\mathrm{K} 372-\mathrm{Me}}$ in the presence of resveratrol compared with the DMSO-treated controls. Importantly, the function of resveratrol towards p53 was attenuated in the presence of shRNA against SET7/9 (Fig. 2D-F). To further confirm the present results, the expression of p53 target genes (PUMA and BAX) were examined using a Luciferase assay (Fig. 3A-D). Treating cells with resveratrol significantly induced PUMA and BAX expression in all cell lines compared with the untreated control cells $(\mathrm{P}<0.05)$, whereas the effect of resveratrol was significantly abolished in the absence of SET7/9 ( $<<0.05$; Fig. 3A-D). Altogether, these results suggest that SET7/9 functions as a key mediator of resveratrol-dependent p53 activation in colorectal cancer cells.

Resveratrol promotes apoptosis through the induction of SET7/9 expression. To identify the physiological role of resveratrol in colorectal cancer cells, the expression of cleaved PARP, an apoptosis biomarker (26,27), was examined in various colorectal cancer cell lines (HCT116, CO115 and SW48 As mentioned in Fig. 1B, a viability test was performed to identify the maximal inhibitory concentration of resveratrol for HCT116, CO115, and SW480 cells, which were 25,30 , and $37.5 \mu \mathrm{M}$ respectively. Therefore, each cell line was treated with a specific
Table I. Association of SET7/9 expression and clinicopathological features in $55 \mathrm{CRCs}$.

\begin{tabular}{|c|c|c|}
\hline Clinicopathological features & Total cases & P-value \\
\hline Age (years) & $62.23 \pm 3.43$ & \\
\hline$\leq 60$ & $21(38.2)$ & $>0.05$ \\
\hline$>60$ & $34(61.6)$ & \\
\hline \multicolumn{3}{|l|}{ Sex } \\
\hline Female & $30(54.5)$ & $>0.05$ \\
\hline Male & $25(45.5)$ & \\
\hline \multicolumn{3}{|l|}{ Tissue } \\
\hline Tumour & 55 & \\
\hline Normal & 55 & \\
\hline \multicolumn{3}{|l|}{ Depth of infiltration } \\
\hline Submucosa, musculeris propria & $14(25.5)$ & $>0.05$ \\
\hline Subserosa & $31(56.4)$ & \\
\hline To the surrounding tissue & $10(18.2)$ & \\
\hline \multicolumn{3}{|l|}{ Histological differentiation } \\
\hline Well differentiated & $8(14.5)$ & $<0.01$ \\
\hline Moderately differentiated & $30(54.5)$ & \\
\hline Poorly differentiated & $17(31.0)$ & \\
\hline \multicolumn{3}{|l|}{ TNM staging ${ }^{\mathrm{a}}$} \\
\hline 0 & 0 & $<0.01$ \\
\hline I & $25(45.5)$ & \\
\hline II & $22(40.0)$ & \\
\hline III & $8(14.5)$ & \\
\hline \multicolumn{3}{|l|}{ Lymph node metastasis } \\
\hline Yes & $32(58.2)$ & 0.0293 \\
\hline No & $23(41.8)$ & \\
\hline
\end{tabular}

a Stage I: tumor size $\leq 2 \mathrm{~cm}$ and no nodal involvement; stage II: tumour size $\leq 2 \mathrm{~cm}$ and 1-3 nodes, or 2-4 $\mathrm{cm}$ and 0-3 nodes, or $4+\mathrm{cm}$ and 0 nodes; stage III: tumour size $\leq 2 \mathrm{~cm}$ and $4+$ nodes, or $2-4 \mathrm{~cm}$ and $4+$ nodes, or $>4 \mathrm{~cm}$ and $1+$ nodes. TNM, tumor node metasitasis: CRC, colorectal cancer.

concentration of resveratrol followed by Western blotting. The results suggested the overexpression of p53 and cleaved PARP in the presence of resveratrol (Fig. 4A-C). Additionally, the ablation of SET7/9 decreased the expression of cleaved PARP upon treatment with resveratrol compared with untransfected cells (Fig. 4D-F). Similar results were obtained when the expression of cleaved caspase-3 was examined (Fig. 4D-F). Altogether, these data confirm that SET7/9 is the mediator of resveratrol-dependent apoptosis in colorectal cancer.

\section{Discussion}

SET7/9, a SET domain-containing lysine methyltransferase, has been proposed to transfer a methyl group to the target lysine residue of non-histone proteins including p53 (methylated at K372) and histone H3 Lysine 4 (H3-K4) (6). Thus, SET7/9 is involved in various cellular pathways in cancer cells, including cell proliferation, cell death, migration and invasion (24). In the present study, SET7/9 was identified as 

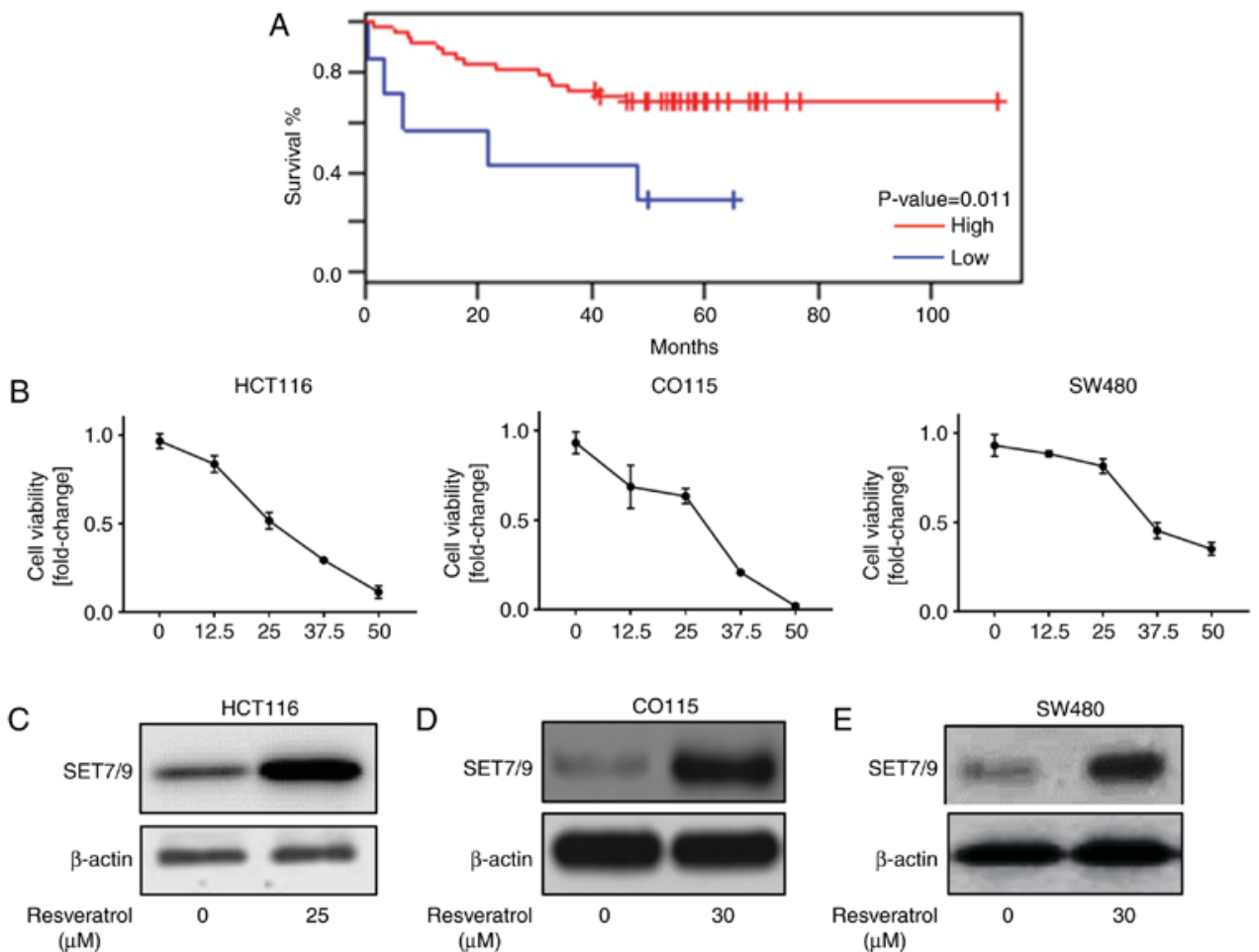

Figure 1. SET7/9 is modulated by resveratrol. (A) Association between SET7/9 expression and the overall survival rates of patients with colorectal cancer. It was determined that a high expression of SET7/9 is significantly associated with better overall survival rates using Kaplan-Meier survival curves ( $\mathrm{P}=0.011)$. This data was obtained from 55 patients. (B) Three colorectal cancer cell lines including HCT116, CO115 and SW48 were cultured and treated with resveratrol $(0-50 \mu \mathrm{M})$ for $24 \mathrm{~h}$ followed by the cell viability being measured using a CellTiter-Glo Luminescent kit. Western blotting revealed the protein expression of SET7/9 in (C) HCT116, (D) CO115 and (E) SW48 cells in response to resveratrol treatment. SET7/9, SET domain containing lysine methyltransferase 7/9.
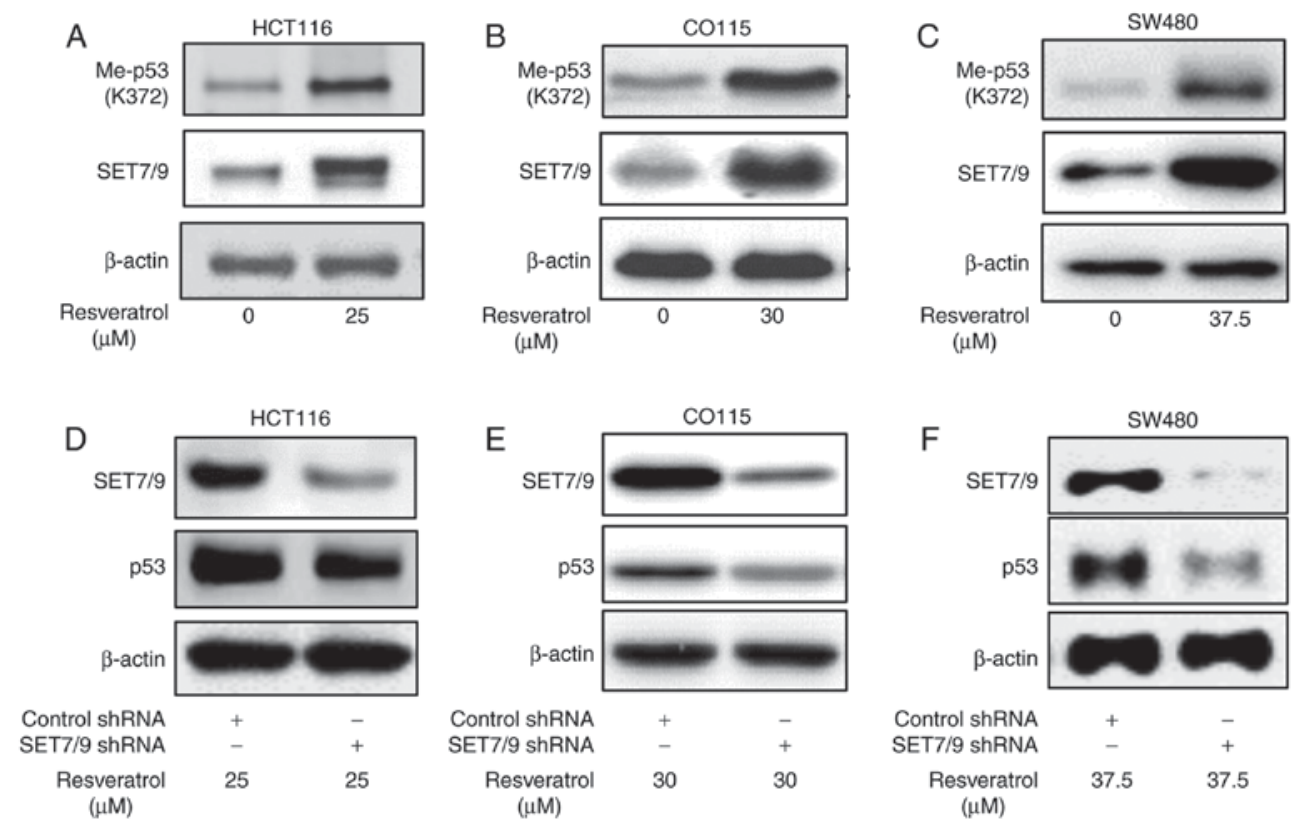

Figure 2. SET7/9 is essential for resveratrol-driven p53 overexpression. Western blotting for (A) HCT116, (B) CO115 and (C) SW48 cells, revealing the expression of Me-p53 at K372 in the presence of resveratrol. Western blotting analysis presenting p53 expression in response to resveratrol in SET7/9-silenced (D) HCT116, (E) CO115 and (F) SW48 cells. SET7/9, SET domain containing lysine methyltransferase 7/9; Me-, methylated; shRNA, short hairpin RNA; K372, lysine 372; p53, tumour protein p53.

a mediator of the resveratrol-driven overexpression of $\mathrm{p} 53$, while the specific molecular mechanisms underlying the interaction between resveratrol and SET7/9 remain unclear. Further experiments are required in order to determine whether resveratrol directly or indirectly promotes SET7/9 expression.

p53 mono-methylation by SET7/9 is crucial for its stabilization and promoter occupancy (28). The results revealed and 

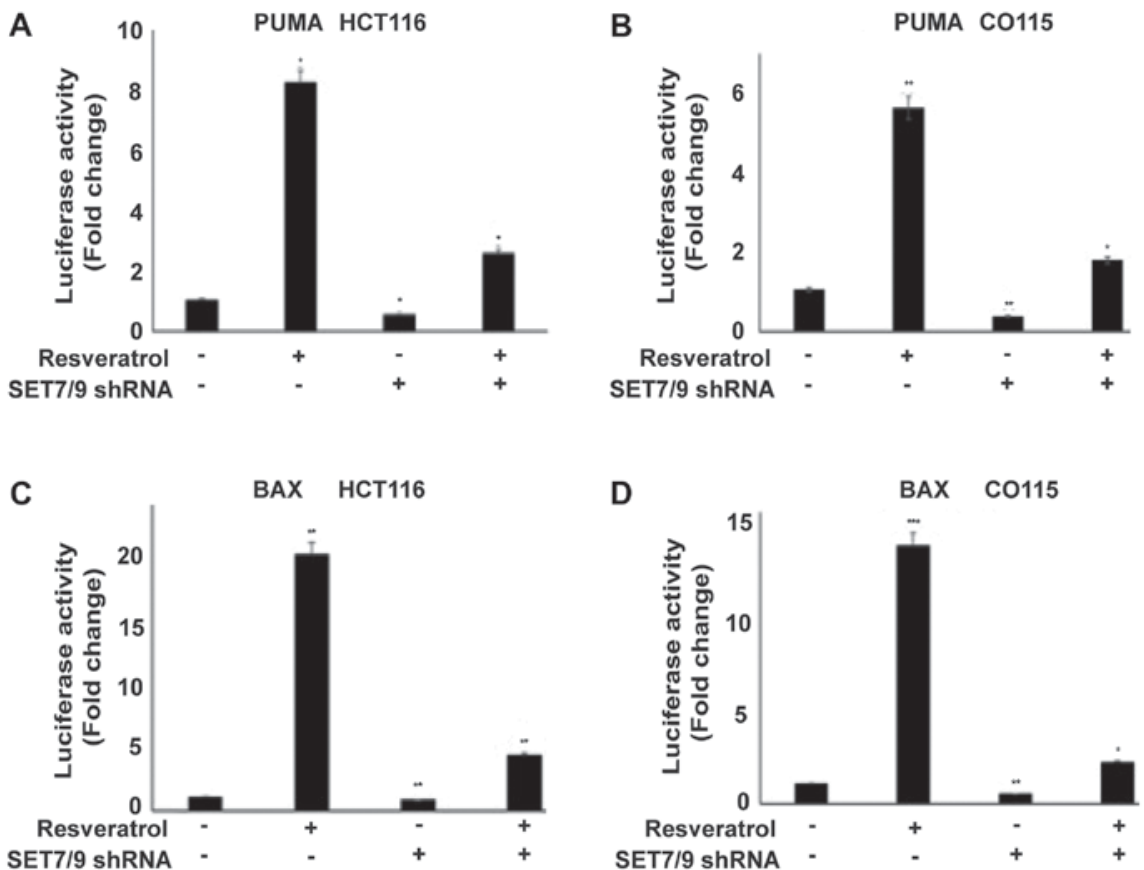

Figure 3. Resveratrol augments p53 activity mainly through the upregulation of SET7/9. PUMA luciferase activity (fold change) in (A) HCT116 and (B) CO115 cells and BAX luciferase activity (fold change) in (C) HCT116 and (D) CO115 cells followed by treatment with resveratrol in the presence or absence of shRNA against SET7/9. Results are the mean of three independent experiments. Error bars represent the standard error of the mean. ${ }^{*} \mathrm{P}<0.05,{ }^{* * *} \mathrm{P}<0.01$ and ${ }^{* * * *} \mathrm{P}<0.001$. SET7/9, SET domain containing lysine methyltransferase 7/9; PUMA, Bcl2 binding component 3; BAX, Bcl2 associated X, apoptosis regulator; shRNA, short hairpin RNA.

A

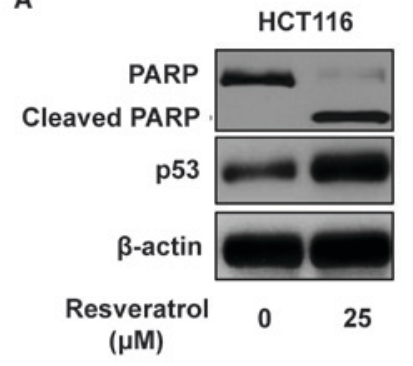

D

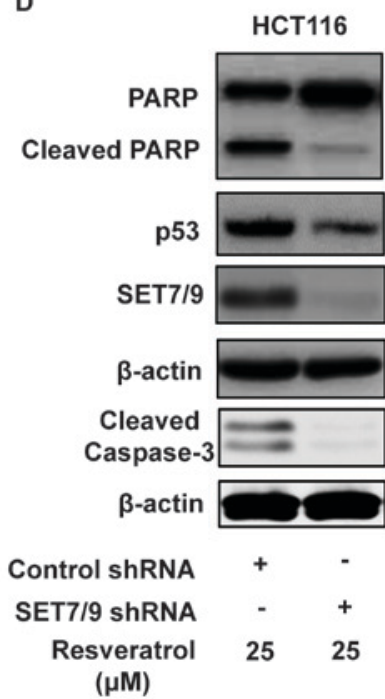

B

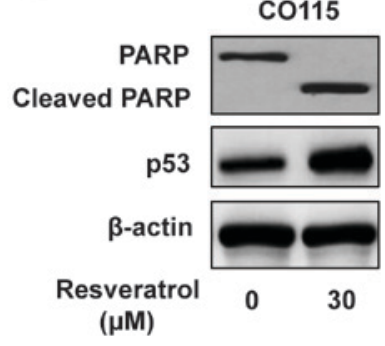

$\mathbf{E}$

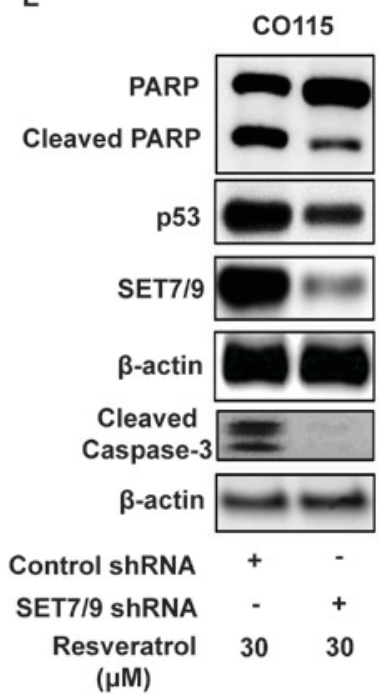

C

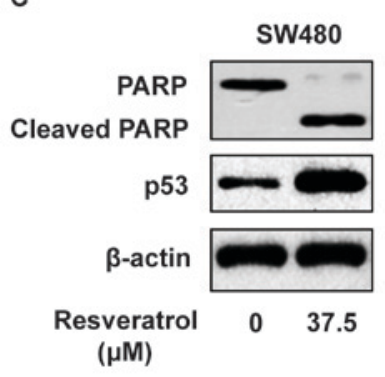

$\mathrm{F}$

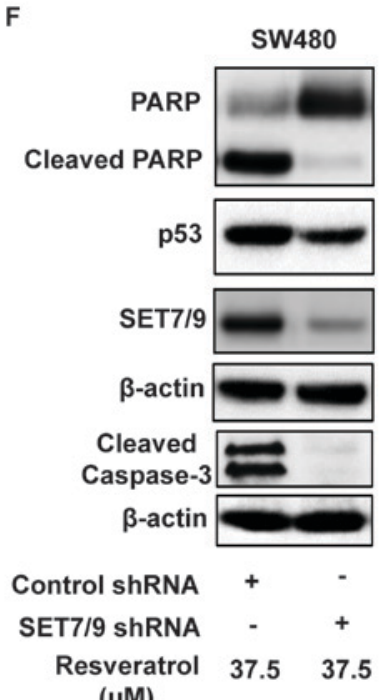

Figure 4. SET7/9 is required for the induction of p53-mediated apoptosis by resveratrol. Western blotting was conducted to evaluate PARP cleavage, apoptosis biomarker, in (A) HCT116, (B) CO115 and (C) SW480 colorectal cancer cells. Western blots revealing the response of SET7/9-depleted (D) HCT116, (E) CO115 and (F) SW480 colorectal cancer cells to resveratrol, revealing the expression of PARP, cleaved PARP, p53, SET7/9 and cleaved caspase-3. p53, tumour protein p53; PARP, poly (ADP-ribose) polymerase; SET7/9, SET domain containing lysine methyltransferase 7/9; shRNA, short hairpin RNA. 


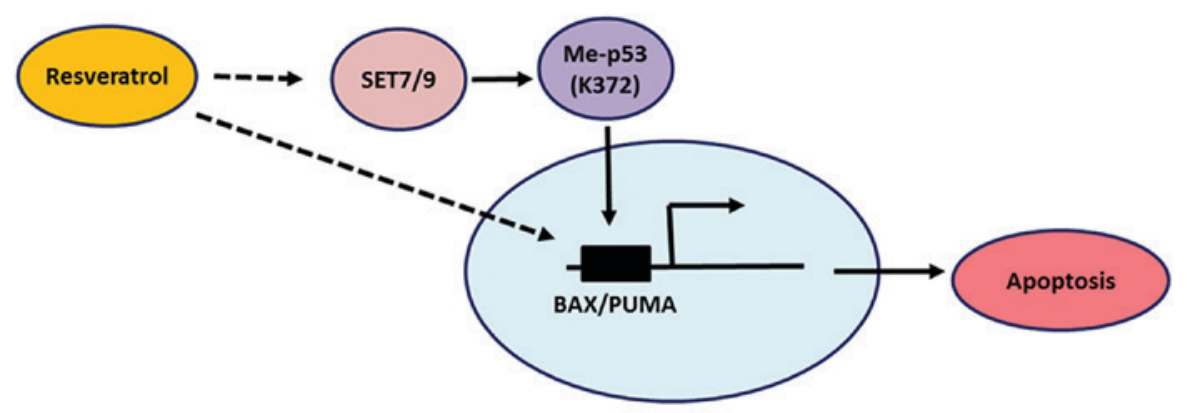

Figure 5. Schematic model of the function of resveratrol in colorectal cancer cells. Resveratrol induces the expression of SET7/9 using an unknown mechanism. SET7/9 methylates p53 at K372 and promotes the p53 transcriptional activity on the PUMA and BAX promoter, which results in apoptosis. In addition to the p53-dependent pathway, resveratrol augments PUMA and BAX expression by activating an unknown signalling pathway. SET7/9, SET domain containing lysine methyltransferase 7/9; Me-, methylation; p53, tumor protein p53; K372, lysine 372; PUMA, Bcl2 binding component 3; BAX, Bcl2 associated X, apoptosis regulator.

upregulation of SET7/9 in colorectal cancer (HCT116, CO115, and SW480) cells upon resveratrol treatment. In agreement with previous studies (16,29-31), increasing SET7/9 led to the induction of the expression of methylated p53 at K372, in addition to the induction of total p53 and p53-target genes. The present study also revealed that resveratrol-induced p53 overexpression was reduced in cells expressing shRNA against SET7/9 compared with untransfected cells. However, resveratrol treatment may still induce the expression of p53 target genes (BAX and PUMA) in SET7/9-depleted cells implying that resveratrol regulates p53 activity by other mechanisms. Further studies are warranted to elucidate the exact molecular mechanisms by which resveratrol regulates p53 expression and activity.

Resveratrol has been reported to be one of the most promising anticancer agents isolated from natural products that induce apoptosis in certain cancer types in a p53-dependent manner (17-20,32). Similarly, the present results propose resveratrol as a positive regulator of apoptosis in colorectal cancer cells (Fig. 4). Furthermore, the data produced by the present study indicates that the pro-apoptotic function of resveratrol depends on SET7/9 in colorectal cancer.

From the present study, it was concluded that SET7/9 functions as a mediator of resveratrol-dependent p53 activation, and the results confirm that resveratrol modulates p53 through its mono-methylation at K372 (Fig. 5). In agreement with the present study, other studies have reported the induction of p53 phosphorylation at serine 15 by resveratrol (21), which is vital for the stabilization and activation of p53 $(11,14,16)$. The results of the present study add another layer of complexity to the anti-cancer function of resveratrol and further studies are required to identify the effect of resveratrol-induced SET7/9 in p53 mutant cancer cells.

\section{Acknowledgments}

The authors would like to thank Dr Chao Gao (Albany Medical College, Albany, NY, USA) for assistance with the bioinformatics analysis

\section{Funding}

The present study was supported by the Lianyungang Health and Family Planning Commission (grant no. 201623).

\section{Availability of data and materials}

The publicly available bioinformatics data set (GSE17537: 244653_at) was downloaded on prognoscan website by this article $(22,23)$ and made publicly available (https://www.ncbi. nlm.nih.gov/geo/query/acc.cgi?acc $=$ GSE17537), which shows overall survival rate.

\section{Authors' contributions}

ZL, XW and JL performed experiments, analyzed data, and wrote the manuscript. HS performed the experiments and wrote the manuscript. FZ supervised the project, designed the experiments, and wrote the manuscript.

\section{Ethics approval and consent to participate}

Not applicable.

\section{Patient consent for publication}

Not applicable.

\section{Competing interests}

The authors declare that they have no competing interests.

\section{References}

1. Singh UP, Singh NP, Singh B, Hofseth LJ, Price RL, Nagarkatti M and Nagarkatti PS: Resveratrol (trans-3,5,4'-trihydroxystilbene) induces silent mating type information regulation-1 and down-regulates nuclear transcription factor-kappaB activation to abrogate dextran sulfate sodium-induced colitis. J Pharmacol Exp Ther 332: 829-839, 2010.

2. Kimura Y and Okuda H: Resveratrol isolated from Polygonum cuspidatum root prevents tumor growth and metastasis to lung and tumor-induced neovascularization in Lewis lung carcinoma-bearing mice. J Nutr 131: 1844-1849, 2001.

3. Schmidlin L, Poutaraud A, Claudel P, Mestre P, Prado E, Santos-Rosa M, Wiedemann-Merdinoglu S, Karst F, Merdinoglu D and Hugueney P: A stress-inducible resveratrol $\mathrm{O}$-methyltransferase involved in the biosynthesis of pterostilbene in grapevine. Plant Physiol 148: 1630-1639, 2008.

4. Das DK and Maulik N: Resveratrol in cardioprotection: A therapeutic promise of alternative medicine. Mol Interv 6: 36-47, 2006. 
5. Colin D, Gimazane A, Lizard G, Izard JC, Solary E, Latruffe N and Delmas D: Effects of resveratrol analogs on cell cycle progression, cell cycle associated proteins and 5fluoro-uracil sensitivity in human derived colon cancer cells. Int J Cancer 124: 2780-2788, 2009.

6. Bai Q, Shen Y, Yao X, Wang F, Du Y, Wang Q, Jin N, Hai J, Hu T and Yang J: Modeling a new water channel that allows SET9 to dimethylate p53. PLoS One 6: e19856, 2011.

7. Ji Q, Liu X, Fu X, Zhang L, Sui H, Zhou L, Sun J, Cai J, Qin J, Ren $\mathbf{J}$ and Li Q: Resveratrol inhibits invasion and metastasis of colorectal cancer cells via MALAT1 mediated Wnt/ $\beta$-catenin signal pathway. PLoS One 8: e78700, 2013

8. Siegel RL, Miller KD, Fedewa SA, Ahnen DJ, Meester RGS, Barzi A and Jemal A: Colorectal cancer statistics, 2017. CA Cancer J Clin 67: 177-193, 2017.

9. Aoki K and Taketo MM: Adenomatous polyposis coli (APC): A multi-functional tumor suppressor gene. J Cell Sci 120 3327-3335, 2007.

10. Losi L, Luppi G and Benhattar J: Assessment of K-ras, Smad4 and $\mathrm{p} 53$ gene alterations in colorectal metastases and their role in the metastatic process. Oncol Rep 12: 1221-1225, 2004.

11. Zilfou JT and Lowe SW: Tumor suppressive functions of $\mathrm{p} 53$. Cold Spring Harb Perspect Biol 1: a001883, 2009.

12. Rada M, Althubiti M, Ekpenyong-Akiba AE, Lee KG, Lam KP, Fedorova O, Barlev NA and Macip S: BTK blocks the inhibitory effects of MDM2 on p53 activity. Oncotarget 8: 106639-106647, 2017.

13. Rada M, Vasileva E, Lezina L, Marouco D, Antonov AV Macip S, Melino G and Barlev N: Human EHMT2/G9a activates p53 through methylation-independent mechanism. Oncogene 36: 922-932, 2017.

14. Bieging KT, Mello SS and Attardi LD: Unravelling mechanisms of p53-mediated tumour suppression. Nat Rev Cancer 14: 359-370, 2014

15. Lee DH, Kim C, Zhang L and Lee YJ: Role of p53, PUMA, and Bax in wogonin-induced apoptosis in human cancer cells. Biochem Pharmacol 75: 2020-2033, 2008.

16. Marouco D, Garabadgiu AV, Melino G and Barlev NA: Lysine-specific modifications of p53: A matter of life and death? Oncotarget 4: 1556-1571, 2013.

17. Cong P, Yi C and Wang XY: Expression of Smo in pancreatic cancer CD $44^{+} \mathrm{CD} 24^{+}$cells and construction of a lentiviral expression vector to silence Smo. Oncology Lett 16: 4855-4862, 2018.

18. Hsieh TC, Wang Z, Hamby CV and Wu JM: Inhibition of melanoma cell proliferation by resveratrol is correlated with upregulation of quinone reductase 2 and p53. Biochem Biophys Res Commun 334: 223-230, 2005.

19. Oi N, Yuan J, Malakhova M, Luo K, Li Y, Ryu J, Zhang L, Bode AM, Xu Z, Li Y, et al: Resveratrol induces apoptosis by directly targeting Ras-GTPase-activating protein $\mathrm{SH} 3$ domain-binding protein 1 . Oncogene 34: 2660-2671, 2015.

20. Li B, Hou D, Guo H, Zhou H, Zhang S, Xu X, Liu Q, Zhang X, Zou Y, Gong Y and Shao C: Resveratrol sequentially induces replication and oxidative stresses to drive p53-CXCR2 mediated cellular senescence in cancer cells. Sci Rep 7: 208, 2017.
21. Shih A, Davis FB, Lin HY and Davis PJ: Resveratrol induces apoptosis in thyroid cancer cell lines via a MAPK- and p53-dependent mechanism. J Clin Endocrinol Metab 87: 1223-1232, 2002.

22. Mizuno H, Kitada K, Nakai K and Sarai A: PrognoScan: A new database for meta-analysis of the prognostic value of genes. BMC Med Genomics 2: 18. 2009

23. Smith JJ, Deane NG, Wu F, Merchant NB, Zhang B, Jiang A, Lu P, Johnson JC, Schmidt C, Bailey CE, et al: Experimentally derived metastasis gene expression profile predicts recurrence and death in patients with colon cancer. Gastroenterology 138: 958-968, 2010.

24. Akiyama Y, Koda Y, Byeon SJ, Shimada S, Nishikawaji T, Sakamoto A, Chen Y, Kojima K, Kawano T,Eishi Y, et al: Reduced expression of SET7/9, a histone mono-methyltransferase, is associated with gastric cancer progression. Oncotarget 7: 3966-3983, 2016.

25. Montenegro MF, Sánchez-Del-Campo L, González-Guerrero R, Martínez-Barba E, Piñero-Madrona A, Cabezas-Herrera J and Rodríguez-López JN: Tumor suppressor SET9 guides the epigenetic plasticity of breast cancer cells and serves as an early-stage biomarker for predicting metastasis. Oncogene 35: 6143-6152, 2016.

26. Los M, Mozoluk M, Ferrari D, Stepczynska A, Stroh C, Renz A, Herceg Z, Wang ZQ and Schulze-Osthoff K: Activation and caspase-mediated inhibition of PARP: A molecular switch between fibroblast necrosis and apoptosis in death receptor signaling. Mol Biol Cell 13: 978-988, 2002.

27. Althubiti M, Rada M, Samuel J, Escorsa JM, Najeeb H, Lee KG, Lam KP, Jones GD, Barlev NA and Macip S: BTK modulates p53 activity to enhance apoptotic and senescent responses. Cancer Res 76: 5405-5414, 2016.

28. West LE and Gozani O: Regulation of p53 function by lysine methylation. Epigenomics 3: 361-369, 2011.

29. Chuikov S, Kurash JK, Wilson JR, Xiao B, Justin N, Ivanov GS, McKinney K, Tempst P, Prives C, Gamblin SJ, et al: Regulation of p53 activity through lysine methylation. Nature 432: 353-360, 2004.

30. Kurash JK, Lei H, Shen Q, Marston WL, Granda BW, Fan H, Wall D, Li E and Gaudet F: Methylation of p53 by Set7/9 mediates p53 acetylation and activity in vivo. Mol Cell 29: 392-400, 2008.

31. Nishioka K, Chuikov S, Sarma K, Erdjument-Bromage H, Allis CD, Tempst P and Reinberg D: Set9, a novel histone H3 methyltransferase that facilitates transcription by precluding histone tail modifications required for heterochromatin formation. Genes Dev 16: 479-489, 2002.

32. Kai L, Samuel SK and Levenson AS: Resveratrol enhances p53 acetylation and apoptosis in prostate cancer by inhibiting MTA1/NuRD complex. Int J Cancer 126: 1538-1548, 2010.

This work is licensed under a Creative Commons Attribution-NonCommercial-NoDerivatives 4.0 International (CC BY-NC-ND 4.0) License. 